\title{
Perfil dos pacientes amputados por acidentes automobilísticos
}

\author{
Profile of patients amputed by automobile accidents
}

\author{
Weidson Pablo Marques Diniz ${ }^{1}$, Adriana Sousa Rego ${ }^{1}$, Tatiana Cristina Fonseca Soares de \\ Santana ${ }^{1}$, José Newton Lacet Vieira ${ }^{2}$
}

Resumo: Objetivo: Descrever o perfil epidemiológico e clínico de pacientes amputados por acidente automobilístico. Materiais e métodos: Tratou-se de um estudo descritivo, transversal, que foi realizado em um hospital público de São José de Ribamar - MA, verificando prontuários no período de janeiro e julho de 2016. Os dados foram coletados através de uma ficha para obter dados sociodemográficos e características clínicas da amputados. Resultados: Foram 63 prontuários de amputados inseridos no estudo, sendo a mediana de idade de 33 anos, com mínimo de 17 anos e máximo de 77 anos. Quanto ao sexo, a amostra teve $81 \%$ do sexo masculino e $19 \%$ do sexo feminino. A amostra constou-se de 63 prontuários. Quanto ao uso de bebida alcóolica, $(76 \%)$ ingeriam bebida alcóolica e $(24 \%)$ não ingeriam. O nível de amputação, mas recorrente foi transfemural com $(30,2 \%)$, transtibial $(20,6 \%)$. A precedência da amputação foi: acidente por colisão $(78 \%)$, atropelamento $(16 \%)$ e queda de moto $(16 \%)$. A maioria $(65 \%)$ da amostra sofreu fratura. Conclusão: Concluiu-se que houve um predomínio de amputações transfemural decorrentes principalmente por traumas decorrentes de colisão no trânsito.

Palavras-chave: Amputação. Acidente automobilístico. Trauma.

Abstract: Objective: to describe the epidemiological and clinical profile of patients amputees by car accident. Materials and methods: this was a descriptive transversal study which was carried out in a public hospital in São José de Ribamar-MA, checking charts in the period January to July 2016. The data were collected through a plug to obtain demographic data and clinical characteristics of amputees. Results: 63 charts of amputees entered into the study, being the median of the age of 33 years, with a minimum of 17 years and a maximum of 77 years. As for sex, the sample had $81 \%$ of males and $19 \%$ of females literate. The sample consisted of 63 records. As the use of liquor, $(76 \%)$ drank less liquor and $(24 \%)$ did not interfered. The level of amputation, but applicant was transfemural with $(30.2 \%)$, $(20.6 \%)$ transtibial. The precedence of the amputation was: collision accident $(78 \%)$, trampling $(16 \%)$ and motorcycle crash (16\%). The majority (65\%) of the sample suffered fracture. Conclusion: It was concluded that there was a predominance of transfemoral amputations mainly due to traumas due to collision in traffic.

Keywords: Amputation. Automobile accident. Trauma.

\footnotetext{
${ }^{1}$ Departamento de Fisioterapia. Universidade Ceuma

${ }^{2}$ Departamento de Fisioterapia. Universidade Ceuma. Endereço para correspondência: Avenida Nova Iorque, Q-5 E, C-03, Central Parque, Araçagy, São José de Ribamar-MA. CEP: 65.110-000. Fone: (98) 981109633.
} 
Introdução

As grandes cidades do mundo perpassaram nas últimas décadas por um acelerado processo de urbanização. Através do advento da revolução tecnológica, houve uma reorganização do ambiente urbano em torno do trânsito e da popularização dos veículos motorizados. ${ }^{1}$ Porém, com a ampliação do fluxo de automóveis, observou-se também um aumento proporcional no número de agentes nocivos a integridade física dos indivíduos, dentre eles, pode-se destacar a deficitária mobilidade urbana, poluição ambiental e acidentes de trânsito. ${ }^{2}$

A Organização Mundial da Saúde ${ }^{3}$, através de estudo realizado no ano de 2012, contatou dados que apontam a ocorrência de 3 a 4 mil óbitos diários decorrentes de acidentes de trânsito no mundo. Em meados da década de 2000, devido a sua gravidade e repercussões, acidentes de trânsito passaram a ser um problema de saúde pública em países em desenvolvimento. ${ }^{4}$

Acidentes de trânsito são eventos que apresentam múltiplas repercussões as vítimas e podem variar conforme inúmeros fatores, desde as características físicas do acidente a mecanismos de segurança utilizados pelos usuários, dentre estes o cinto de segurança e o capacete de motociclistas são apontados como aspectos protetores a lesões mais graves. $^{5}$

Ao se investigar os fatores causais dos acidentes automobilísticos, constatou que em $90 \%$ dos acidentes registrados no país, as causas estão relacionadas à falha humana, baseada nos princípios de negligência, imprudência ou imperícia, como o ato de dirigir sob efeito do álcool ou abuso de velocidade. ${ }^{6}$
A epidemiologia dos acidentes de transito tem sido delineada através de estudos que constatam a tendência de vítimas do gênero masculino e em idade jovem. ${ }^{7,8}$ De acordo com estudo realizado por Abreu et al. ${ }^{9}$ este dado é justificado devido a homens jovens se exporem mais a situações de risco, como dirigir em alta velocidade, realizar manobras arriscadas ou fazerem uso do veículo para causar boa impressão, bem como o uso de bebidas alcoólicas entre esta faixa etária ser mais comum. Malta et al. ${ }^{5}$ alertam que o risco é grave não apenas para os usuários dos automóveis que estão expostos a colisões entre os veículos, como também para pedestres e ciclistas, susceptíveis a atropelamentos.

Os dados preocupantes residem não tão somente na quantidade de óbitos produzidas por estes acidentes, mas também na quantidade de sobreviventes que sofreram lesões que na maioria das vezes geram repercussões tanto no âmbito individual, quanto para a coletividade, visto que indivíduos com incapacidades temporárias ou permanentes tendem a uma perda significativa de qualidade de vida e produção econômica. ${ }^{9}$

Os tipos de lesões mais prevalentes de acidentes de trânsito são contusões $(64,6 \%)$, escoriações $(51,1 \%)$, cortes $(22,7 \%)$ e fraturas fechadas (14,7\%). Quanto a localização das lesões em vítimas de acidentes, as fraturas nos membros inferiores são mais prevalentes, todavia, o índice elevado de trauma crânio encefálico deve ser considerado, bem como o desfecho de amputação. ${ }^{10}$

Amputação é definida como a secção de uma estrutura corporal de forma permanente com o objetivo de impedir o avanço de um sinal clínico patológico, implica na perda de autonomia do paciente e dificuldade para realizar atividades de vida diária. ${ }^{11}$ As amputações decorrentes de 
acidentes de trânsito têm como causa fisiopatológica mais frequente afecções vasculares periféricas, podendo também derivar de causas neurológicas ou de necrose tecidual. ${ }^{12}$

Estudo realizado por Dornelas ${ }^{13}$, exclusivamente com indivíduos amputados por decorrência de acidente de trânsito, verificou que os níveis de secção mais frequentes foram os transfemurais, em que ocorre a secção de parte da estrutura óssea chamada fêmur e transtibiais, em que a estrutura seccionada é a tíbia.

A cidade de São Luís, possui uma frota de 366.633 mil veículos circulando diariamente nas ruas, ${ }^{14}$ ambos representando um risco potencial para o desfecho de acidentes de trânsito e consequente danos a integridade física de condutores e pedestres. Neste contexto, a implementação de pesquisas que abordem o universo das amputações decorrentes de acidentes de trânsito e seus fatores associados tornam-se imprescindíveis para a proposição de políticas tanto de prevenção, quanto de abordagem ao paciente amputado em sua integralidade, bem como na obtenção de um panorama acerca dos números de amputações decorrentes de acidentes. Diante do exposto, esse estudo objetivou descrever o perfil epidemiológico e clínico de pacientes amputados por acidente automobilístico.

\section{Materiais e métodos}

Tratou-se de um estudo descritivo, transversal, realizado sobre o perfil sociodemográfico e as características clínicas de pessoas amputadas, com base em dados coletados em prontuários do Hospital de Urgência e Emergência Clementino Moura - Socorrão II, localizado na
Avenida Tancredo Neves, nº 3685, São José de Ribamar. Maranhão.

Amostra foi constituída por 63 prontuários de pacientes que sofreram algum tipo de amputação devido ao acidente automobilístico. Foram incluídas fichas de indivíduos de ambos os sexos com idade entre 17 e 74 anos de idade, registrados entre janeiro e julho de 2016. E como o critério de exclusão foram fichas de indivíduos com desfecho de óbito, e o processo de reamputação.

As variáveis estudadas foram: idade, sexo, escolaridade, situação conjugal, consumo de bebida alcóolica, e nível de amputação.

O estudo foi aprovado pelo Comitê de Ética em Pesquisa em Humanos (CEP) da Universidade Ceuma, com 0 parecer $\mathrm{n}^{\circ}$ $628.878 / 2014$.

Os dados foram analisados no SPSS 18.0. Na analise descritiva, as variáveis qualitativas são descritas em frequência absoluta e relativa e as quantitativas em mediana, mínimo e máximo.

\section{Resultados e Discussão}

Dentre os 63 amputados inseridos no estudo, a mediana da idade foi de 33, sendo o mínimo 17 e o máximo 74 anos (gráfico 1).

A maioria dos amputados pertence ao sexo masculino, residem em São Luís e são solteiros (tabela 1). Observou-se um número de 63 amputados, predominatemente jovens nascidos em São Luís com idade mediana de 33 anos, a maior exposição masculina é devido à maior circulação urbana de automóveis dessa faixa etária.

A predominância do sexo masculino descrita nessa média de idade corresponde ao descrito no estudo de Cavalcante et al. ${ }^{15} \mathrm{diz}$ que 
esta maior vulnerabilidade encontrada na população jovem, é devido ao envolvimento de jovens nos acidentes de trânsito estar relacionado a imaturidade, no que se refere a detecção e prevenção da possibilidade de conflitos no trânsito, e o excesso de autoconfiança na condução de veículo. O estudo indica o crescente índice de acidentes automobilístico de homens solteiros $(66,7 \%)$ em São Luís, com $(41,3 \%)$ possuindo escolaridade de nível fundamental, e a raça autoinformada foi à negra com (42,9\%). As características sociodemográficas de amputados citadas evidencia um público que se destaca, urgindo a necessidade de maior conscientização, pois mesmo após as aprovações das leis que proíbem uso de álcool para condutores de automóveis, as taxas de amputados e taxas de óbitos ainda continuam a aumentar.

Em relação aos hábitos de vida, $(76 \%)$ das pessoas que tiveram amputação, relataram uso de bebida alcoólica (gráfico 2), entretanto, apenas
$22,2 \%$ (14) referem uso de cigarro. O estudo relata que $76 \%$ faziam uso de bebida alcoólica e $24 \%$ não ingeriam álcool. Seguidos de outros hábitos como uso de cigarro que influencia junto ao consumo de bebidas de forma a ser prejudicial e de risco à saúde do indivíduo.

São utilizados como meio auxiliar de mobilização social: Propagandas educativas, palestras estaduais e municipais visando práticas educativas no trânsito e orientações ao cidadão para comportamento no trânsito. ${ }^{16}$

Entre os participantes do estudo, o nível de amputação mais verificado foi o transfemural (gráfico 3). Conforme os dados supracitados além de a amputação transfemural (30,2\%), outras amputações comuns em acidentes deste tipo, o estudo descreve que as amputações transtibial (20,6\%) e do pododáctilo (19\%) também apresentam níveis de porcentagem elevada dentro da população pesquisada.

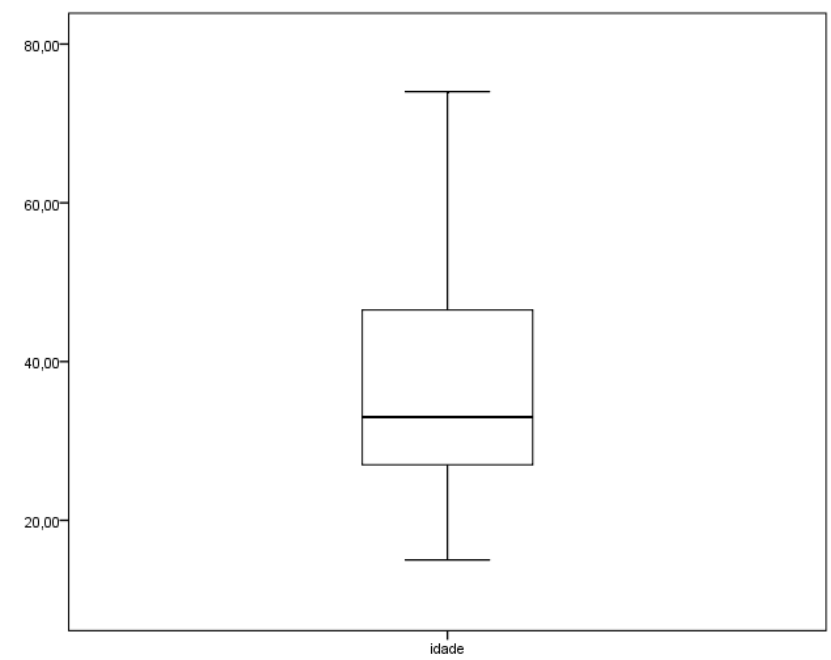

Gráfico 1-Idade dos amputados por acidente automobilístico. São Luís - MA, 2016. 
Tabela 1- Características sociodemográficas de amputados por acidente automobilístico. São Luís -MA, 2016.

\begin{tabular}{lcc}
\hline \multicolumn{1}{c}{ Características } & Frequência absoluta & Frequência relativa \\
\hline \multicolumn{1}{c}{ Sexo } & 12 & $19,0 \%$ \\
Feminino & 51 & $81,0 \%$ \\
$\begin{array}{l}\text { Masculino } \\
\quad \text { Escolaridade }\end{array}$ & 47 & $74,6 \%$ \\
Fundamental & 16 & $25,4 \%$ \\
Médio & & \\
$\quad$ Estado civil & 42 & $66,7 \%$ \\
Solteiro & 21 & $33,3 \%$ \\
Casado & & \\
$\quad$ Raça & 27 & $42,9 \%$ \\
Negro & 25 & $39,7 \%$ \\
Pardo & 4 & $6,3 \%$ \\
Branco & 7 & $11,1 \%$ \\
Não informado & & \\
$\quad$ Naturalidade & 36 & $57,1 \%$ \\
São Luís & 23 & $36,5 \%$ \\
Interior do Maranhão & 1 & $1,6 \%$ \\
Outros Estados & 3 & $4,8 \%$ \\
Não informado & & $7,9 \%$ \\
$\quad$ Ocupação & 5 & $4,8 \%$ \\
Estudante & 3 & $33,3 \%$ \\
Aposentado & 21 & $54,0 \%$ \\
Outras profissões & 34 & \\
Não informado & &
\end{tabular}

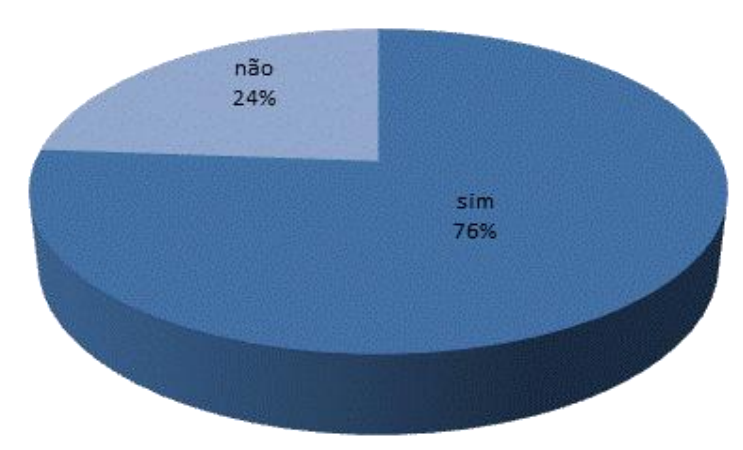

Gráfico 2-Uso de bebida alcoólica em amputados por acidente automobilístico. São Luís - MA, 2016. 


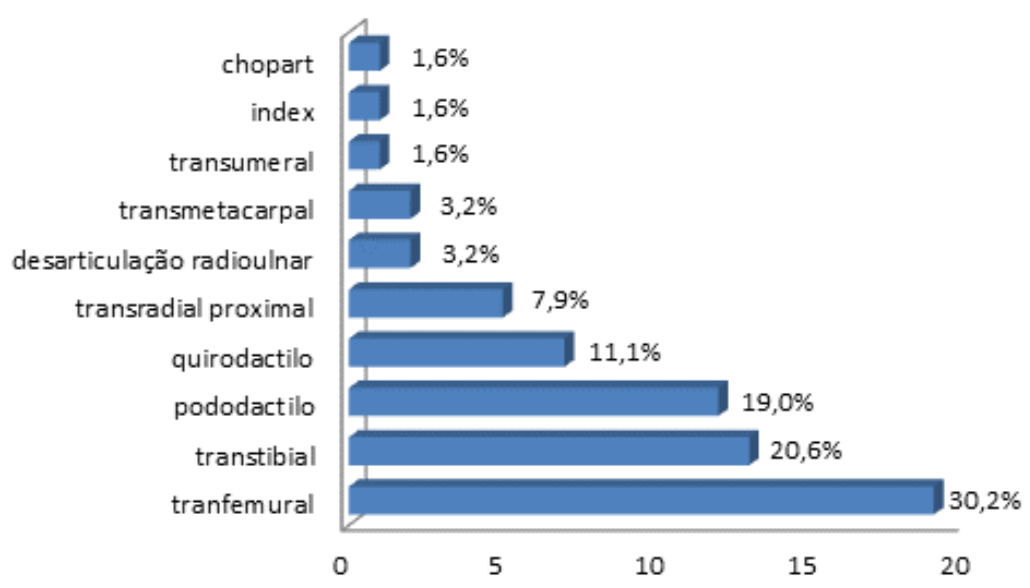

Gráfico 3-Nível de amputação de indivíduos que sofreram acidente automobilístico. São Luís - MA, 2016.

A amputação transfemural consiste em uma incisão circular que deve ser realizada mediante manipulação delicada e envolve dor pós-operatória, envolve complexa sutura dos músculos e principais troncos arteriais e vasos femorais a serem ligados. ${ }^{17}$

A amputação transtibial possui a característica de longo retalho posterior, são evitadas necroses por meio preciso desse retalho formado por músculos gastrocnêmios e solear. ${ }^{18}$ Amputação de pododáctilo significa amputação de membro inferior (pé), o maior perigo que envolve incisão nessa área é a ocorrência de infecção ou isquemia do tecido na aplicação após da incisão de retalho medial ou lateral. ${ }^{1}$

Referente ao tipo de acidente que ocasionou a amputação, $77,8 \%$ foi por colisão (gráfico 4). Entre os veículos envolvidos nesses acidentes, $87 \%$ (55) são motocicleta (55) e $13 \%$ (8) automóveis.

Bôas \& Silva ${ }^{19}$ consideram que se configura como atropelamento, acidentes que afetam pessoas que estão transitando em via pública, as consequências do atropelamento além do risco de amputação pela periculosidade podem desencadear a morte. Estudo de Dornelas ${ }^{13}$, sobre o uso de prótese salienta que para quem sobrevive 0 atropelamento e tem que amputar resta reabilitação que se coloca como uma fase difícil levando à depressão e frustração.

Estudo realizado por Golias et al. ${ }^{20}$ sobre a caracterização e custos de acidentes de motocicleta, afirmou que a colisão entre veículos apontado como $78 \%$ envolve acidente entre ambos, quando se encontram em velocidade em uma mesma direção, podem ser vários os danos causados pela colisão as consequências podem levar à amputação quanto maior a velocidade, maior o risco de danos à saúde.

Apesar de ser apontado no estudo com menor porcentagem a queda de moto $(6 \%)$ também envolve grande risco de amputação, a queda de moto ainda gera maior risco pela instabilidade do veículo, ou seja, maior facilidade em gerar acidentes.

Além da amputação, $61 \%$ (41) dos participantes do estudo também 


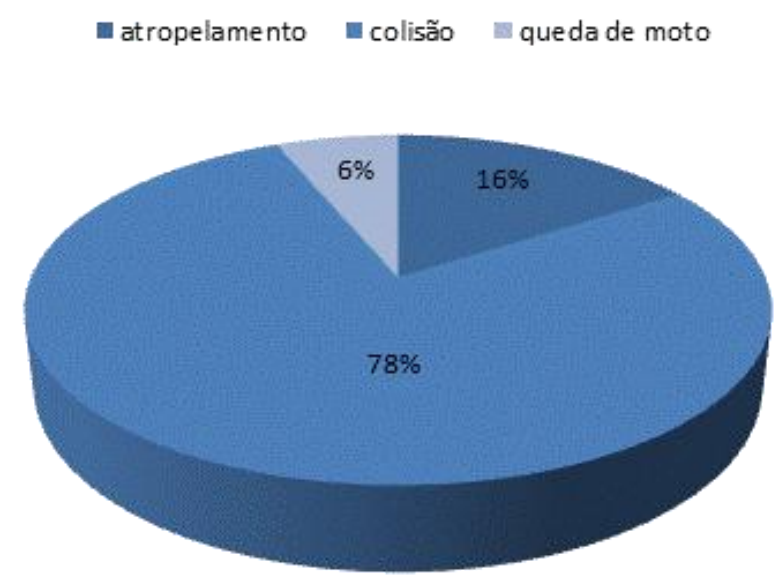

Gráfico 4 -Tipo de acidente dos amputados por acidente automobilístico. São Luís MA, 2016.

sofreram fratura (gráfico 5). Entre esses, $70 \%$ (28) foi nos membros inferiores e $30 \%$ (13) nos membros superiores.

Observa-se que além da amputação os acidentados ainda tiveram fraturas, traumatismos que podem atingir órgãos vitais. A gravidade do acidente acomete ao adoecimento e uma recuperação lenta, incapacitando o indivíduo a exercer suas atividades do cotidiano, o repouso se torna extremamente importante para o avanço da restauração da qualidade de vida. ${ }^{21}$

Lacerda et al. ${ }^{22}$ expõem que a maior parte dessas fraturas são expostas, o que evidência estado de dor, propiciando em vários até a deficiência física. Quando ocorrem tais acidentes o indivíduo passa por período de readaptação a uma nova vida que envolve incapacidade de recuperação rápida.

O gráfico 6 apresenta a média de dias internados dos amputados que foi de 4 dias, com mínimo de 1 dia e no máximo 57 dias. Vale ressaltar que o paciente internado em decorrência do procedimento cirúrgico evoluir para inúmeras sequelas funcionais nas suas atividades de vida diária, que influencia na recuperação do individuo amputado. O apoio familiar é imprescindível e deve ser estimulado para haver base sólida que estimule a recuperação. ${ }^{22}$

Desta forma o tempo de internação está diretamente ligado à natureza das lesões do paciente amputado, são fatores preponderantes o tipo de tratamento, condições de saúde e estado psicológico e emocional. ${ }^{23}$ 


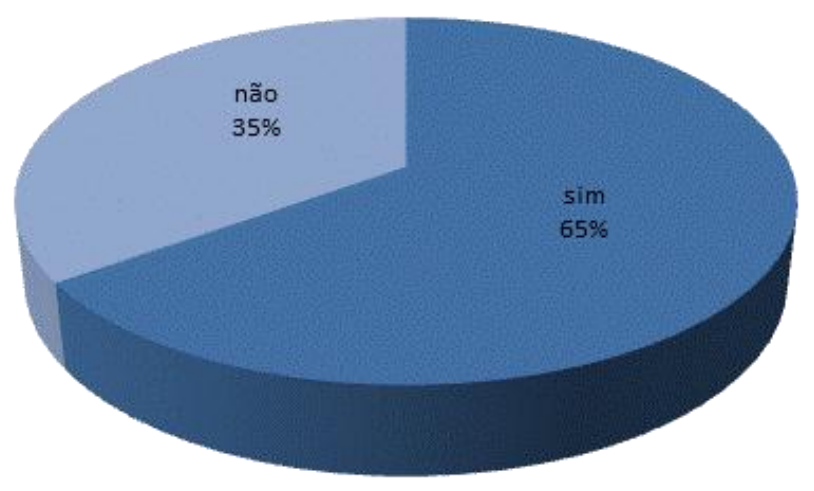

Gráfico 5 - Fratura em amputados por acidente automobilístico. São Luís - MA, 2016.

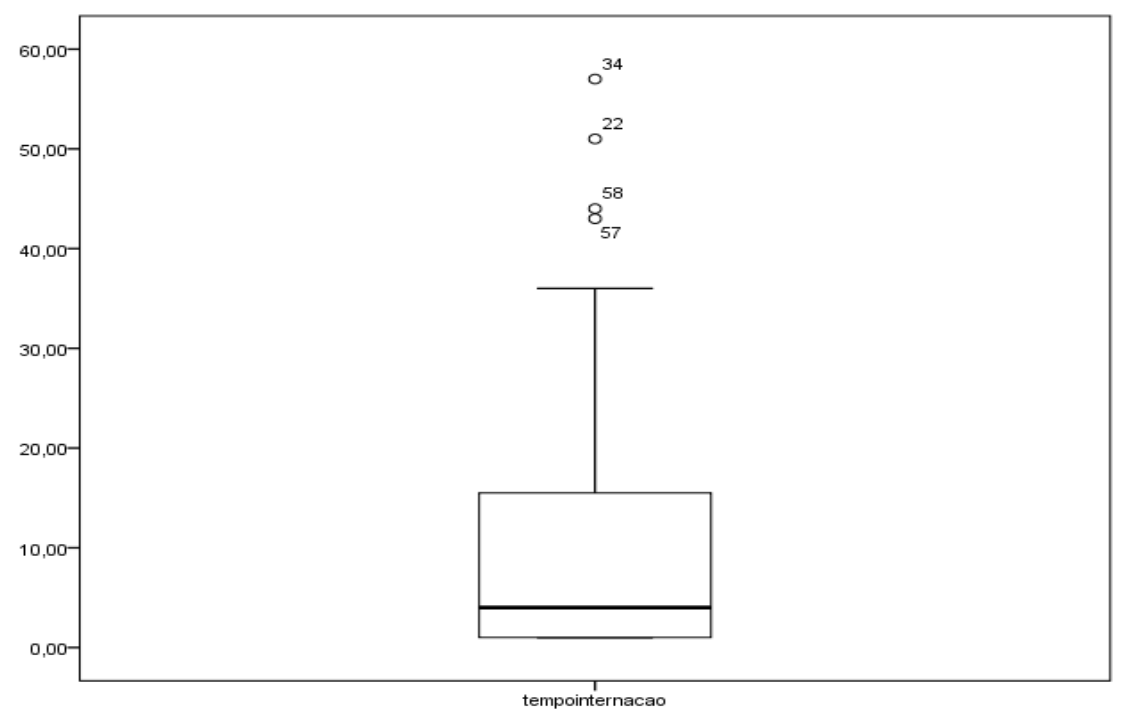

Gráfico 6 - Dias de internação de amputados por acidente automobilístico. São Luís MA, 2016. 


\section{Conclusão}

O estudo sobre 0 perfil epidemiológico e clínico de pacientes amputados por acidentes automobilísticos mostrou que a maior parte dos acidentes que ocasionaram a amputação ocorreu por colisão no trânsito. O estudo mostrou que a maioria das pessoas sofreram fraturas, em membros inferiores.

Recomenda-se que sejam realizados outros estudos sobre 0 tema, para obtenção de dados comparativos, devido o número crescente de amputados ocasionados por acidentes automobilísticos, assim, a sociedade urge na necessidade de debater sobre o assunto para que por meio da conscientização haja uma ação preventiva que evite indicadores de saúde negativos relacionados ao perfil epidemiológico e clínico de pacientes.

\section{Referências}

1. Luccia N, Silva ES. Amputações dos membros inferiores [Internet]. Franca: Concurso e Fisioterapia; 2012 [acesso 2016 nov 11]. Disponível em: http://www.concursoefisioterapia.com/ 2012/05/amputacoes-dos-membrosinferiores.html.

2. Bacchieri G, Barros AJD. Acidentes de trânsito no Brasil de 1998 a 2010: muitas mudanças e poucos resultados. Rev Saúde Pública. 2011;45(5):949-63.

http://dx.doi.org/10.1590/S003489102011005000069.

3. Organização Mundial de Saúde. Mortality and global health estimates. Geneva: World Health Organization; 2012.

4. Nardi ACF, Malta DC, Duarte E, Garcia LP. Segurança no trânsito: tempo de resultados. Epidemiol Serv Saúde. 2015;24(4):593-4.
http://dx.doi.org/10.5123/S1679-

49742015000400001.

5. Malta DC, Mascarenhas MDM, Bernal RTI, Silva MMA, Pereira CA, Minayo MCS, et al. Análise das ocorrências das lesões no trânsito e fatores relacionados segundo resultados da Pesquisa Nacional por Amostra de Domicílios (PNAD) Brasil. Ciênc Saúde Coletiva. 2011;16(9):3679-87. http://dx.doi.org/10.1590/S141381232011001000005.

6. Macêdo DW, Oliveira FPA. Epidemiologia de acidentes urbanos com atendimento móvel de urgência, Santarém, PA, maio a setembro de 2009. Rev Saúde Desenvolv. 2012;2(1):107-26.

7. Caixeta $\mathrm{CR}$, Minamisava $\mathrm{R}$, Oliveira LMAC, Brasil VV. Morbidade por acidentes de transporte entre jovens de Goiânia, Goiás. Ciênc Saude Coletiva. 2010;15(4):2075-84. http://dx.doi.org/10.1590/S141381232010000400021.

8. Carvalho RC, Costa MCO, Silva JJM, Rebouças MC. Acidentes de trânsito envolvendo adolescentes: o registro da situação de Feira de Santana, Bahia, em 2001. Rev Baiana Saúde Pública. 2011;29(1):22-34.

9. Abreu AMM, Lima JMB, Matos LN, Pillon SC. Uso de álcool em vítimas de acidentes de trânsito: estudo do nível de alcoolemia. Rev Latino-Am Enfermagem. 2010;18(6):513-20. http://dx.doi.org/10.1590/S010411692010000700005.

10. Silva Filho CAR, Reis ES, Barros IGP. Perfil das pacientes vítimas de acidente de trânsito, atendidos na clínica de fisioterapia da Unic no ano de 2005 a 2008. Uniciências. 2010;14(1):83-94.

http://dx.doi.org/10.17921/1415-

5141.2010v14n1p\%25p.

11. Macêdo MCM, Chamlian TR, Leal CAP, Bonilha MMM, Rezende F. Retorno ao trabalho de pacientes com amputação traumática de membros inferiores. Acta Fisiátr. 2013;20(4):179-82. 
http://dx.doi.org/10.5935/01047795.20130029 .

12. Santos LF, Fritzen PG, Gonçalves BR, Melo SA, Silva VF. Perfil das amputações de membros inferiores de pacientes cadastrados na associação de deficientes físicos de Apucarana. Saúde e Pesquisa. 2010;3(1):59-64.

13. Dornelas LF. Uso da prótese e retorno ao trabalho em amputados por acidentes de transporte. Acta Ortop Bras. 2010;18(4):204-6. http://dx.doi.org/10.1590/S141378522010000400006.

14. Departamento Estadual de Trânsito do Maranhão. Estatística de veículos. São Luís; 2016 [acesso 20 mar. 2016]. Disponível em: http://servicos.detran.ma.gov.br/Estati sticas/Veiculo.

15. Cavalcante AKCB, Hoalanda VM, Rocha CFM, Cavalcante SW, Sousa JPR, Sousa FHR. Perfil dos acidentes de trânsito atendidos por serviço préhospitalar móvel. Rev Baiana Enferm. 2015;29(2):135-45.

http://dx.doi.org/10.18471/rbe.v29i2.1 2656.

16. Dias CA. Álcool e direção: a influência do uso do álcool na condução de veículos automotores. Maceió: Universidade Paulista Curso de Especialização em Psicologia do Trânsito; 2013.

17. Rodrigues NB, Gimenes CM, Lopes $\mathrm{CM}$, Rodrigues JMS. Mortes, lesões e padrão das vítimas em acidentes de trânsito com ciclomotores no município de Sorocaba, São Paulo, Brasil. Rev Fac Ciênc Méd. 2010;12(3):21-5.

18. Araújo RA, Andrade PKFL, Tôrres BR. Principais recursos fisioterapêuticos utilizados em amputados transfemorais durante a fase de pré protetização. In: Anais do XI Encontro de Iniciação à Docência; abr. 9-11 2008; João Pessoa. João Pessoa: UFPB; 2008 [acesso 21 nov 2016]. Disponível em: http://www.prac.ufpb.br/anais/xenex_x ienid/xi_enid/monitoriapet/ANAIS/Area 6/6CCSDFTMT05.pdf.

19. Bôas EFV, Silva MA. Trânsito e segurança pública: impactos e consequências. Humanidades. 2015;4(2):113-28.

20. Golias ARC, Caetano R, Vianna CMM. Caracterização e custos de acidentes de motocicleta com vítimas atendidas em regime de hospitalização no município de Paranavaí-PR no ano de 2007. Physis. 2013;23(4):1123-46. http://dx.doi.org/10.1590/S010373312013000400006.

21. Neves ACM, Mascarenhas MDM, Silva MMA, Malta DC. Perfil das vítimas de violências e acidentes atendidas em serviços de urgência e emergência do Sistema Único de Saúde em capitais brasileiras - 2011. Epidemiol Serv Saúde. 2013;22(4):587-96. http://dx.doi.org/10.5123/S1679 49742013000400005.

22. Lacerda KM, Fernandes RCP, Nobre LCC. Acidentes de trabalho fatais em Salvador, BA: descrevendo o evento subnotificado e sua relação com a violência urbana. Rev Bras Saúde Ocup. 2014;39 (129):63-74. http://dx.doi.org/10.1590/03037657000064812.

23. Chamlian TR, Varanda RR, Pereira CL, Resende JM, Faria CC. Perfil epidemiológico dos pacientes amputados de membros inferiores atendidos no Lar Escola São Francisco entre 2006 e 2012. Acta Fisiatr. 2013;20(4):219-23. http://dx.doi.org/10.5935/01047795.20130036. 